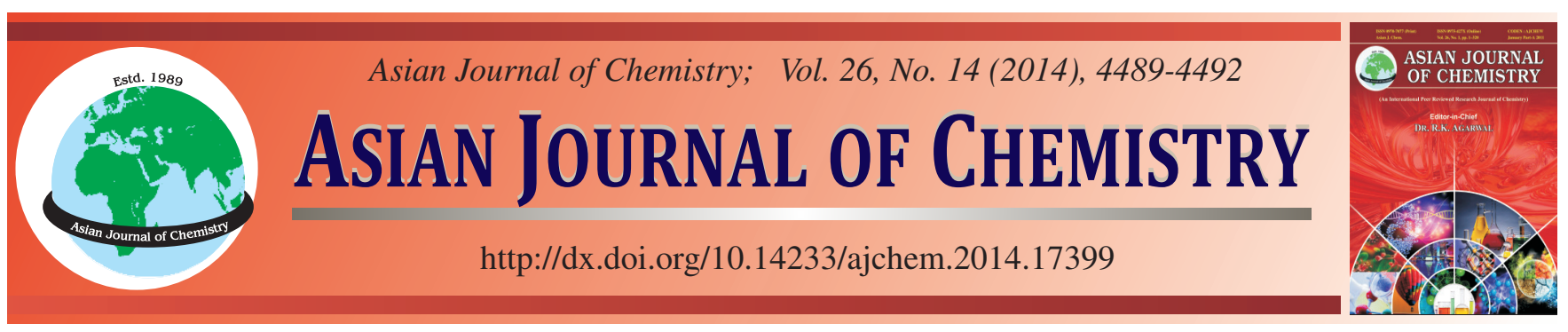

\title{
Optimal Parameters for Preparation of Magnesium Chloride Whisker Composites Using Metal Ion Catalysis Method
}

\author{
Pinghua Zhu ${ }^{1,2, *}$, ShuORAn ZenG ${ }^{2}$ and Lei WANG ${ }^{3}$
}

\begin{abstract}
${ }^{1}$ Jiangsu Institute of Marine Resources, Huaihai Institute of Technology, Lianyungang 222005, P.R. China ${ }^{2}$ Department of Chemical Engineering, Huaihai Institute of Technology, Lianyungang 222005, P.R. China ${ }^{3}$ Nanjing University of Chinese Medicine, Nanjing 210023, P.R. China

*Corresponding author: E-mail: lygzph@163.com
\end{abstract}

Received: 15 February 2014;

Accepted: 15 May 2014;

Published online: 5 July 2014;

AJC-15503

Magnesium chloride purified from bittern is selected as the raw material and the influencing factors of basic magnesium chloride whiskers prepared using a metal ion catalysis method at normal pressure are investigated. The effects of the intensity of metal ions, the reaction temperature and the addition of inoculating seeds on the preparation of magnesium chloride whiskers are studied carefully and the morphology of basic magnesium chloride whiskers is analyzed using a scanning electron microscope. It is found that the optimum parameters for the preparation of basic magnesium chloride whiskers using a metal ion catalysis method at normal pressure are as follows: $\mathrm{Zn}^{2+}$ concentration of $30-60 \times 10^{-6} \mathrm{~mol} / \mathrm{L}$, reaction temperature of $50{ }^{\circ} \mathrm{C}$ and percentage of added inoculating seeds of $0.06 \%$.

Keywords: Magnesium chloride, Whisker, Metal ions, Whisker seeds.

\section{INTRODUCTION}

Seawater resources are abundant in China and the country ranks first in the world in the production of salt from seawater. The amount of bittern produced every year is approximately $2000 \times 10^{4} \mathrm{~m}^{3}$, which contains $392 \times 10^{4} \mathrm{~m}^{3}$ of magnesium chloride. However, the applications of bittern are relatively few $^{1}$. The development of a new type of whisker composite tends to promote the application of all types of seawater bittern and is of considerable significance. The aim of this study is to utilize the solution of magnesium chloride purified from bittern and explore the process conditions of preparing basic magnesium chloride whiskers using a metal ion catalysis method at normal pressure. A basic magnesium chloride whisker is a type of toughening and reinforcing material suitable for plastics, rubbers and ceramics. Magnesium chloride whiskers can work as a flame retardant ${ }^{2-6}$ and a mixture of magnesium chloride whiskers and light calcium carbonate can be used for the combination filling of melt adhesives ${ }^{7,8}$.

\section{EXPERIMENTAL}

Magnesium chloride (prepared from bittern) and analytical reagents: $25 \%$ ammonia water, absolute ethyl alcohol and zinc sulphate.

First, a solution of magnesium chloride is prepared according to requirements and it is poured into a beaker and put in a water bath kettle. The temperature is adjusted to $50{ }^{\circ} \mathrm{C}$. A certain amount of $25 \%$ ammonia water and a solution of metal ions are dropped into the beaker at a constant speed. The reaction time is from $1-2 \mathrm{~h}$ and the solution is stirred at a constant speed. Then, the obtained saturated solution is aged and crystallized in a drying oven at approximately $55{ }^{\circ} \mathrm{C}$ for $24-72 \mathrm{~h}$. The reaction products are washed with distilled water and undergo sucking filtration. Finally, the precipitate is washed with absolute ethyl alcohol for 2-3 times and dried in the drying oven. Thus, magnesium chloride whiskers can be obtained.

\section{RESULTS AND DISCUSSION}

The effects of three factors, namely the concentration of the $\mathrm{Zn}^{2+}$, the reaction temperature and the amount of inoculating seed added, on whisker nucleation are considered. The optimum process of preparing basic magnesium chloride whiskers is determined.

Effects of concentration of metal ion $\mathrm{Zn}^{2+}$ on the growth of magnesium chloride whiskers: The experimental conditions and the results are as shown in Table-1:

The scanning electron microscope (SEM) images of the products are given in Fig. 1a-d and show the crystal morphology of substances from groups A-1 to A-4, respectively.

It is inferred from the results that the effects of the concentration of the metal ion $\mathrm{Zn}^{2+}$ on the formation and the morphology of whiskers are very evident. The changes in the highest 
TABLE-1

EFFECTS OF THE CONCENTRATION OF METAL ION $\mathrm{Zn}^{2+}$ ON THE GROWTH OF MAGNESIUM CHLORIDE WHISKERS

\begin{tabular}{|c|c|c|c|c|c|}
\hline No. & $\begin{array}{c}{\left[\mathrm{Zn}^{2+}\right]} \\
(\mathrm{mol} / \mathrm{L})\end{array}$ & $\begin{array}{c}\mathrm{MgCl}_{2}: \\
\text { aq. } \mathrm{NH}_{3} \\
\text { (molar ratio) }\end{array}$ & $\begin{array}{c}\text { Reactionte } \\
\text { temperature } \\
\left({ }^{\circ} \mathrm{C}\right)\end{array}$ & $\begin{array}{l}\text { Quantity } \\
\text { of crystal } \\
(\mathrm{g})\end{array}$ & $\begin{array}{c}\text { Crystal } \\
\text { morphology }\end{array}$ \\
\hline A-1 & $1 \times 10^{-6}$ & 5 & 40 & 2.6578 & $\begin{array}{l}\text { Acicular, } \\
\text { small size }\end{array}$ \\
\hline A-2 & $45 \times 10^{-6}$ & 5 & 40 & 4.1210 & $\begin{array}{l}\text { Acicular, } \\
\text { medium } \\
\text { size }\end{array}$ \\
\hline A-3 & $90 \times 10^{-6}$ & 5 & 40 & 3.3128 & $\begin{array}{l}\text { Acicular, } \\
\text { big size }\end{array}$ \\
\hline A-4 & $135 \times 10^{-6}$ & 5 & 40 & 2.0385 & $\begin{array}{c}\text { Non- } \\
\text { uniform, } \\
\text { big size }\end{array}$ \\
\hline
\end{tabular}

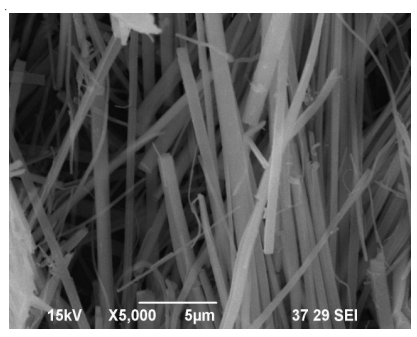

(a)

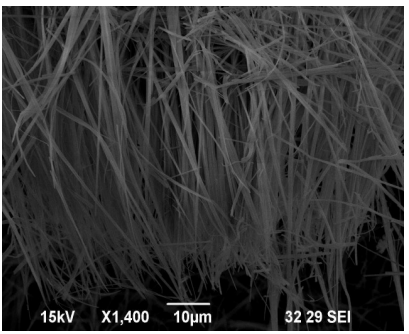

(c)

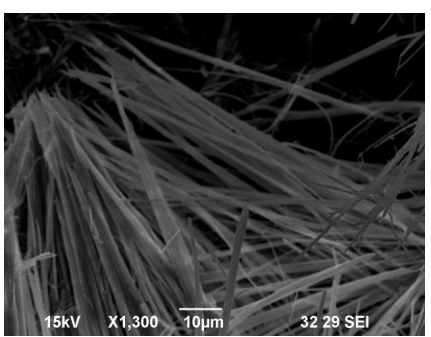

(b)

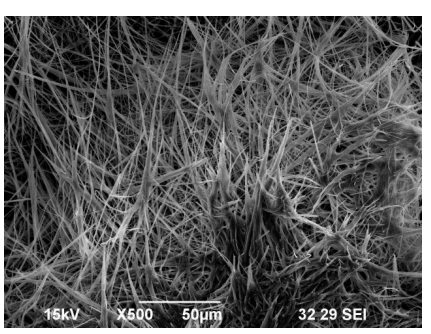

(d)
Fig. 1. SEM pictures of products obtained at different $\mathrm{Zn}^{2+}$ concentrations

draw ratio of whiskers along with the concentration are illustrated in Fig. 2.

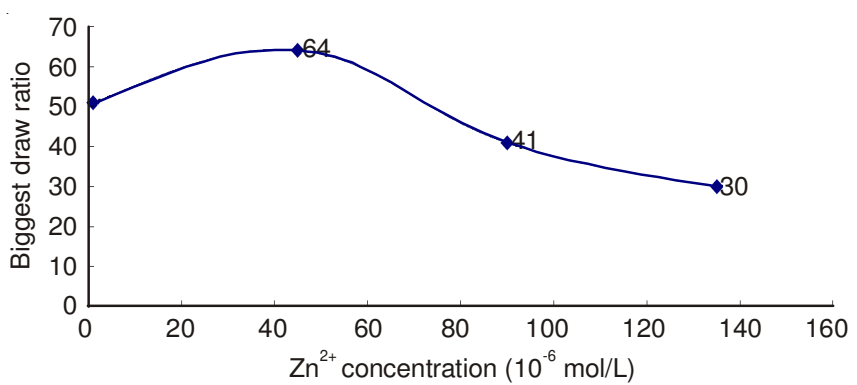

Fig. 2. Schematic representation of the highest draw ratio of whiskers changing with $\mathrm{Zn}^{2+}$ concentration

Fig. 2 showed that the draw ratio of the basic magnesium chloride whiskers changes with an increase in the $\mathrm{Zn}^{2+}$ concentration in the solution and the trend shows a considerable increase at first and then, a decrease at the end. The reason is that $\mathrm{Zn}^{2+}$ can catalyze the formation of whiskers and speed up the growth of whiskers. If the speed of the formation and growth of the whiskers is relatively fast, a thick initial inoculating seed can be formed. When the length of the whiskers increases, the axial growth of the whiskers stops and the cross growth begins. The draw ratio of the growing whiskers is relatively small under these circumstances.

The changes in the yield of the whiskers with respect to the $\mathrm{Zn}^{2+}$ concentration are illustrated in Fig. 3 .

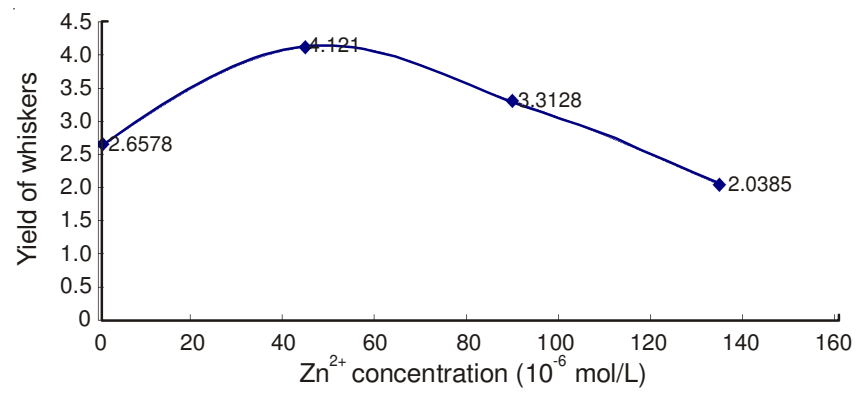

Fig. 3. Changes in the yield of whiskers with respect to $\mathrm{Zn}^{2+}$ concentration

Fig. 3 showed that the output of the basic magnesium chloride whiskers changes with an increase in the $\mathrm{Zn}^{2+}$ concentration in the solution; the trend shows an increase at first and a decrease at the end. This is attributed to the fact that $\mathrm{Zn}^{2+}$ can catalyze the formation of whiskers and speed up the growth of whiskers. The growth is relatively fast and the space for the whiskers' growth is limited, which makes the residual space even smaller, stops the growth of the whiskers and reduces the output of the whiskers. The output of the basic magnesium chloride whiskers changes with an increase in the $\mathrm{Zn}^{2+}$ concentration in the solution. The trend shows an increase at first and then, a decrease at the end. It is given that the $\mathrm{Zn}^{2+}$ concentration is relatively small and the range of change is not wide, the optimum reaction $\mathrm{Zn}^{2+}$ concentration is from 30 to $60 \times 10^{-6}$ $\mathrm{mol} / \mathrm{L}$ according to a comprehensive consideration of the output and the morphology of the whiskers.

Effects of reaction temperature on the growth of magnesium chloride whiskers: We obtain the SEM images of the basic magnesium chloride whiskers under the condition of different temperatures. Further, the experimental results and statistics are presented in Table- 2 .

\begin{tabular}{ccccc}
\multicolumn{5}{c}{ TABLE-2 } \\
\multicolumn{5}{c}{ EFFECTS OF TEMPERATURE ON SYNTHESIZED WHISKERS } \\
\hline No. & {$\left[\mathrm{Zn}^{2+}\right]$} & $\mathrm{MgCl}_{2}$ :aq. $\mathrm{NH}_{3}$ & Reaction & Crystal \\
$(\mathrm{mol} / \mathrm{L})$ & $($ molar ratio) & temp. $\left({ }^{\circ} \mathrm{C}\right)$ & morphology \\
\hline B-1 & $45 \times 10^{-6}$ & 5 & 0 & No product \\
B-2 & $45 \times 10^{-6}$ & 5 & 10 & Small size, impurity \\
B-3 & $45 \times 10^{-6}$ & 5 & 30 & Medium size \\
B-4 & $45 \times 10^{-6}$ & 5 & 50 & Medium size \\
B-5 & $45 \times 10^{-6}$ & 5 & 70 & Dispersed,flake \\
\hline
\end{tabular}

The SEM images of some products obtained from the experiment are shown in Fig. 4a-e represent the crystal morphology of substances from groups $\mathrm{C}-1$ to $\mathrm{C}-5$, respectively.

The temperature is an important influencing factor. According to Fig. 2, when the reaction temperature is low (it is less than $10{ }^{\circ} \mathrm{C}$ as shown in the case of $\mathrm{C}$-1), irregular substances are formed. When the reaction temperature is very high (it is more than $50{ }^{\circ} \mathrm{C}$ as shown in the case of C-5 in Fig. 4), the whiskers of impurity are obtained. According to Fig. 4, the 


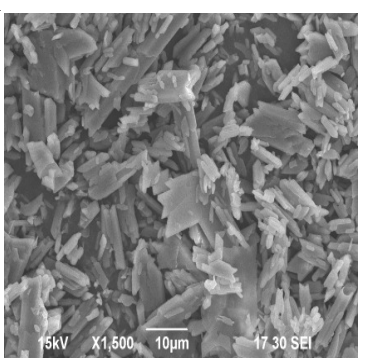

(a)

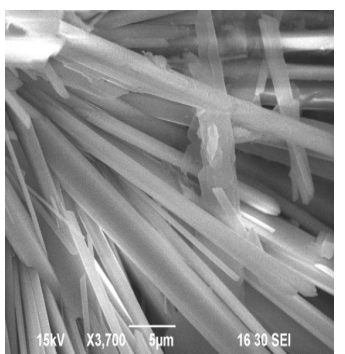

(b)

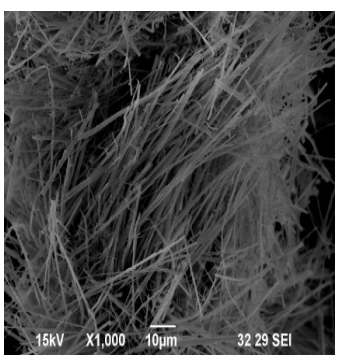

(c)

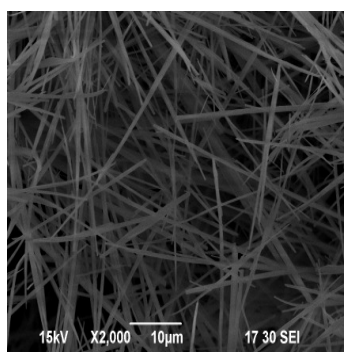

(d)

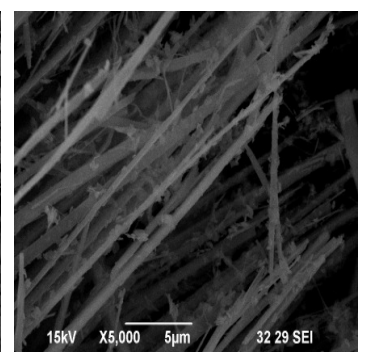

(e)

Fig. 4. SEM images of products obtained at different reaction temperatures

draw ratio of the whiskers is 22 when the reaction temperature is $30^{\circ} \mathrm{C}$. The draw ratio of the whiskers is 35 when the reaction temperature is $40^{\circ} \mathrm{C}, 58$ when the reaction temperature is $50^{\circ} \mathrm{C}$ and 20 when the reaction temperature is $70{ }^{\circ} \mathrm{C}$.

A diagram of whiskers with the highest draw ratio at different temperatures is shown in Fig. 5.

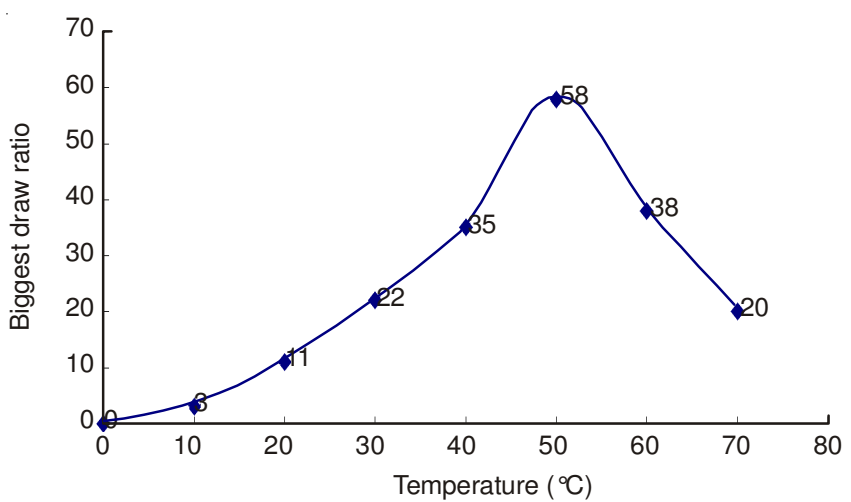

Fig. 5. Diagram of whiskers with the highest draw ratio at different temperatures

Fig. 5 showed that the highest draw ratio of the basic magnesium chloride whiskers changes with an increase in the temperature. The trend shows a gradual increase and then, a decrease at the end. This can be attributed to the fact that an increase in temperature can promote the activity of the reaction system. At a higher reaction temperature, the whisker growth is faster and the initial inoculating seed is thicker. When the length of the whiskers increases, the axial growth of the whiskers stops and the cross growth begins. The draw ratio of the obtained whiskers decreases. Therefore, the optimum reaction temperature is $50{ }^{\circ} \mathrm{C}$.

Effects of addition of inoculating seeds on the growth of basic magnesium chloride whiskers: We obtain the SEM images of the basic magnesium chloride whiskers with different percentages of added whisker seeds; the experimental results and the obtained statistics are given in Table- 3 .

\begin{tabular}{ccccc}
\multicolumn{5}{c}{ TABLE-3 } \\
\multicolumn{5}{c}{ EFFECTS OF ADDING } \\
NHISKER SEEDS ON WHISKER SYNTHESIS \\
No. & $\begin{array}{c}\text { Percentage of } \\
\text { whisker seeds } \\
\text { added }(\%)\end{array}$ & $\begin{array}{c}\text { Reaction } \\
\text { temperature } \\
\left({ }^{\circ} \mathrm{C}\right)\end{array}$ & $\begin{array}{c}\text { Quality of } \\
\text { crystal }(\mathrm{g})\end{array}$ & $\begin{array}{c}\text { Crystal } \\
\text { morphology }\end{array}$ \\
\hline C-1 & 0 & 50 & 2.6578 & Small size \\
C-2 & 0.03 & 50 & 3.0785 & Small size, impurity \\
C-3 & 0.06 & 50 & 4.1213 & Medium size \\
C-4 & 0.09 & 50 & 3.7563 & Medium size \\
C-5 & 0.12 & 50 & 2.0385 & Needle, flake \\
\hline
\end{tabular}

The SEM images of some products obtained from the experiment are shown in Fig. 6a-e and reveal the crystal morphology of substances from groups C-1 to C-5.

According to the above results, the percentage of whisker seeds added has a significant effect on the formation and the morphology of whiskers. The changes in the highest draw ratio with a change in the percentage of the whisker seeds added are shown in Fig. 7.

Fig. 7 showed that the draw ratio of basic magnesium chloride whiskers changes with a change in the percentage of added whisker seeds. The trend shows a gradual increase and then, a decrease at the end. This can be attributed to the fact that an increase in temperature can promote the activity of the reaction system. When the reaction temperature is higher, the whisker growth is faster and the initial inoculating seed is thicker. When the length of the whiskers increases, the axial growth of the whiskers stops and the cross growth begins. The draw ratio of the growing whiskers decreases under these circumstances.

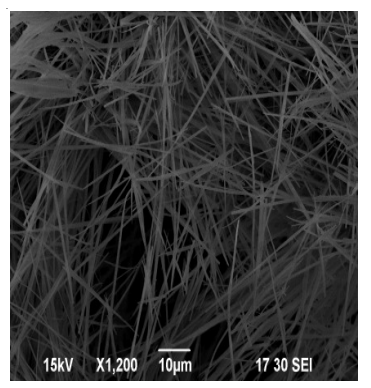

(a)

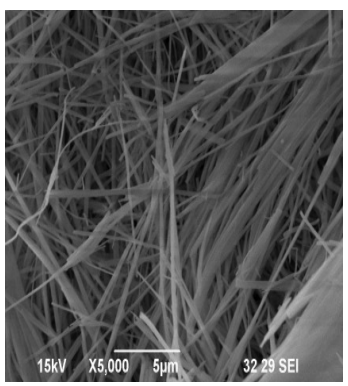

(b)

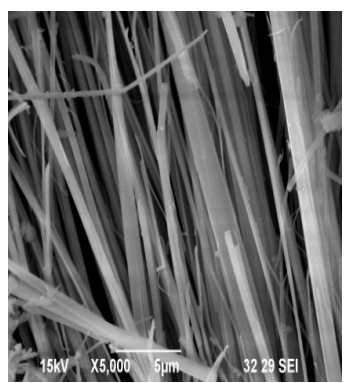

(c)

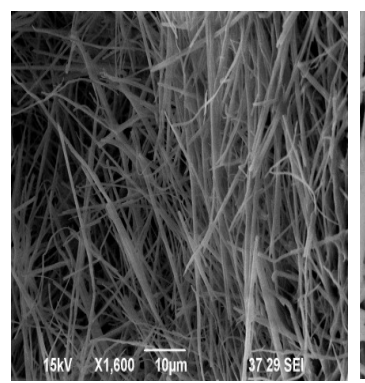

(d)

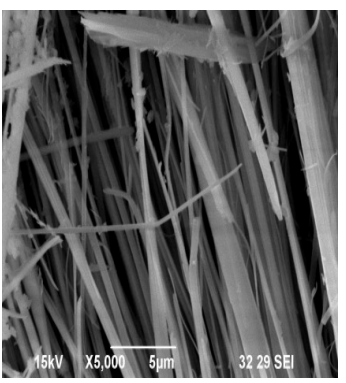

(e)

Fig. 6. SEM images of products obtained upon the addition of whisker seeds 


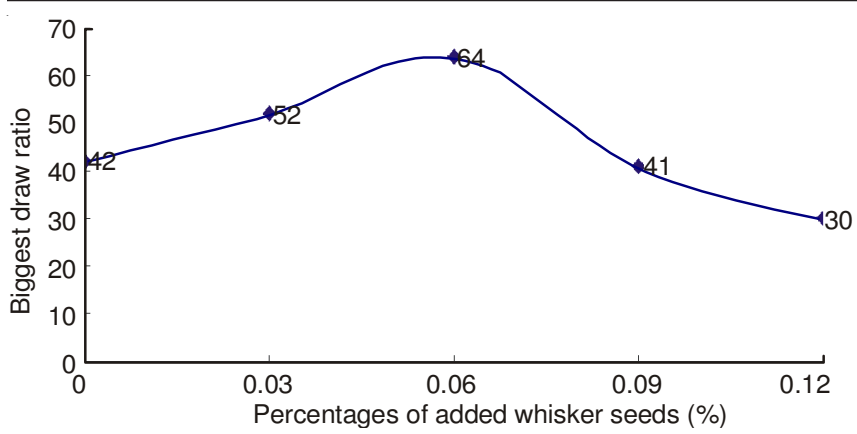

Fig. 7. Draw ratio of whiskers calculated for different percentages of added whisker seeds

The changes in the output brought about by the changes in the percentage of added whisker seeds are shown in Fig. 8.

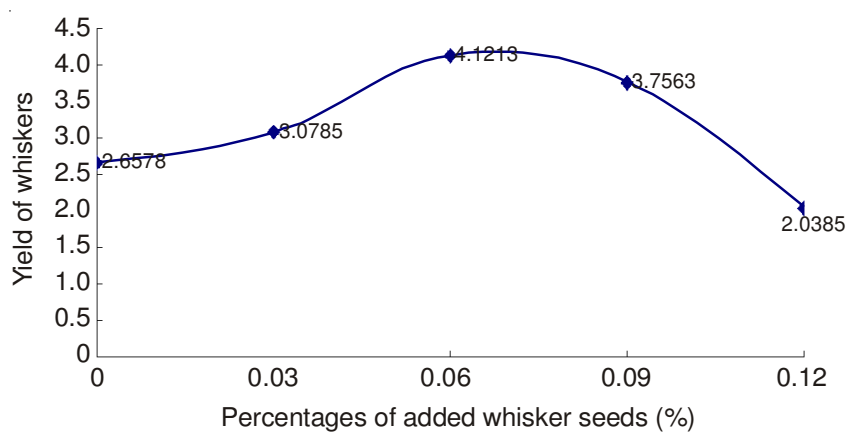

Fig. 8. Output of whiskers obtained for different percentages of added whisker seeds

Fig. 8 showed that the output of the basic magnesium chloride whiskers changes with a change in the percentage of added whisker seeds; the trend shows a significant increase at first and then, a decrease at the end. This can be attributed to the fact that the whisker seeds can catalyze the formation of whiskers. The growth is relatively fast and the space for the whiskers' growth is limited, which makes the residual space even smaller, stops the growth of the whiskers and reduces the output of the whiskers. The optimal percentage of the added whisker seeds is $0.06 \%$ according to a comprehensive consideration of the draw ratio and the output of whiskers.

\section{Conclusion}

The analysis of SEM samples and compared the highest draw ratio of basic magnesium chloride whiskers under different conditions and have considered the coefficient of utilization of materials, energy consumption and output for determining the optimum process of the preparation of basic magnesium chloride whiskers using a metal ion catalysis method. The optimal parameters for this process have been found to be as follows: $\mathrm{Zn}^{2+}$ concentration of $30-60 \times 10^{-6}$ $\mathrm{mol} / \mathrm{L}$, reaction temperature of $50{ }^{\circ} \mathrm{C}$ and the percentage of added whisker seeds of $0.06 \%$.

\section{ACKNOWLEDGEMENTS}

This article is financially supported by Technology openingfund project of Jiangsu Marine Resources Development Institute (JSIMR201301), the Priority Academic Program Development of Jiangsu Higher Education Institutions and 2013 Annual Students' Practical and Innovative Training Program.

\section{REFERENCES}

1. Z.M. Wang, China Non-Met. Mining Ind. Herald, 5, 6 (2006).

2. T.N. Tiegs, L.A. Hamis and P.F. Bether, Am. Ceram. Soc. Bull., 66, 549 (1987).

3. K. Oka, T. Yanagida, K. Nagashima, H. Tanaka and T. Kawai, J. Appl. Phys., 104, 013711 (2008).

4. L. Hu, Y.X. Li, J.P. Qu, Z.X. Huang, X.T. Huang, X.X. Ding, C. Tang and S.R. Qi, Nanosci. Nanotechnol., 4, 1071 (2004).

5. L. Chen, C. Xu and X.-F. Zhang, Mol. Struc. Theochem., 863, 55 (2008).

6. Q. Yang, J. Sha, L. Wang, Y. Wang, X. Ma, J. Wang and D. Yang, Nanotechnology, 15, 1004 (2004).

7. L.D. Zhang, J. Yang and J.S. Yuan, Adhesion China, 10, 25 (2004).

8. J. Li, Y.J. Gao and J.C. Ren, China Pulp Paper Ind., 27, 53 (2003). 